\title{
Melatonin protects against streptozotocin-induced diabetic cardiomyopathy through the mammalian target of rapamycin (mTOR) signaling pathway
}

\author{
Yasemin Behram Kandemir ${ }^{1, A-D}$, Veysel Tosun ${ }^{2, B-D}$, Ünal Güntekin ${ }^{3, E, F}$ \\ ${ }^{1}$ Department of Anatomy, Faculty of Medicine, Harran University, Şanlıurfa, Turkey \\ ${ }^{2}$ Department of Cardiology, Şanlıurfa Education and Research Hospital, Turkey \\ ${ }^{3}$ Department of Cardiology, Faculty of Medicine, Akdeniz University, Antalya, Turkey \\ A - research concept and design; $\mathrm{B}$ - collection and/or assembly of data; $\mathrm{C}$ - data analysis and interpretation; \\ $D$ - writing the article; $E$ - critical revision of the article; $F$ - final approval of the article
}

\section{Address for correspondence}

Yasemin Behram Kandemir

E-mail:ybkandemir07@gmail.com

\section{Funding sources}

None declared

Conflict of interest

None declared

Received on August 3, 2018

Reviewed on 0ctober 2, 2018

Accepted on February 7, 2019

Published online on March 7, 2019

Cite as

Kandemir YB, Tosun V, Güntekin Ü. Melatonin protects against streptozotocin-induced diabetic cardiomyopathy through the mammalian target of rapamycin (mTOR) signaling pathway. Adv Clin Exp Med. 2019;28(9):1171-1177. doi:10.17219/acem/103799

DOI

10.17219/acem/103799

Copyright

Copyright by Author(s)

This is an article distributed under the terms of the Creative Commons Attribution Non-Commercial License (http://creativecommons.org/licenses/by-nc-nd/4.0/)

\begin{abstract}
Background. Several studies demonstrated that the overexpression of mammalian target of rapamycin (mTOR) signaling protein is associated with cardiomyopathy. However, the effect of mTOR on the heart in hyperglycemic condition is still controversial.

Objectives. We aimed to investigate the expression of mTOR and antioxidant enzyme activity in cardiac hypertrophy in rats with streptozotocin-induced diabetes mellitus (DM), and the effects of the melatonin on diabetic cardiomyopathy (DCM).

Material and methods. Forty male Wistar rats were used as the experimental animals. The rats were divided into 4 groups (10 animals in each): group 1 (control group), group 2 (ethanol vehicle group), group 3 (iatrogenically DM-induced group), and group 4 (group given melatonin after iatrogenical DM induction). Streptozotocin was injected intraperitoneally to group 3 and 4 to induce experimental type $1 \mathrm{DM}$. Melatonin was injected intraperitoneally at a dose of $50 \mathrm{mg} / \mathrm{kg} /$ day for 56 days to group 4 . We investigated expression of mTOR levels in heart muscle fibers of all groups. Laboratory analysis and transthoracic echocardiography were performed.
\end{abstract}

Results. Melatonin increased the activity of superoxide dismutase (SOD), catalase (CAT) and glutathione peroxidase (GPX), which were reduced due to hyperglycemia. The mTOR expression levels were significantly higher in group 3 (DM group) compared with controls, whereas melatonin treatment significantly decreased the levels of mTOR expression in group 4 (melatonin + DM group). Diabetic rats developed myocardial hypertrophy with preserved cardiac function.

Conclusions. Cardioprotective effect of melatonin may reduce damages caused by DM in the heart muscle fibers through the mTOR signaling pathway.

Key words: melatonin, diabetic cardiomyopathy, mammalian target of rapamycin 


\section{Introduction}

Left ventricular hypertrophy (LVH) - an increase of left ventricular mass (LVM) - is an independent predictor of cardiovascular disease (CVD). ${ }^{1}$ Diabetic cardiomyopathy (DCM) is one of the common diseases that cause LVH. High glucose-induced alterations in the myocardium structure, including cardiac fibrosis, cardiomyocyte hypertrophy and cardiac microvascular injury, play an important role in the pathophysiology of DCM and lead to the loss of systolic and diastolic function. ${ }^{2-4}$ Intensive glycemic control can reduce microvascular and macrovascular complications of diabetes mellitus (DM), but it has not been fully demonstrated whether there is an obvious cardiovascular and all-cause mortality reduction in DCM. ${ }^{5}$ Therefore, it is very important to carry out experimental studies on the prevention and treatment of DCM.

The mammalian target of rapamycin (mTOR), a member of the PI3K (phosphatidylinositol 3-kinase) related protein kinase family, is an important downstream molecule in the insulin and insulin-like growth factor 1 (IGF-1) signaling pathways, and plays a crucial role in cell growth, metabolism, and cell proliferation. ${ }^{6}$ Previous reports have demonstrated the significant role of mTOR in cardiac function and metabolism in normal and diabetic hearts. ${ }^{7,8}$ Release of free radicals caused by high glucose levels and consequent oxidative stress accelerate lipid peroxidation in muscle fibers, which results in the damage of their normal structure and the disruption of the cell membrane.9,10 Oxidative stress and the mTOR signaling are 2 key factors within the pathological mechanisms of DCM.11,12

Melatonin (N-acetyl-5-methoxytryptamine) is a circadian endocrine molecule secreted by the pineal gland, which has important functions, such as being a powerful antioxidant, anti-apoptotic and free radical purifier. ${ }^{13}$ As was shown in previous studies, melatonin is a highly effective antioxidant that can prevent the harmful effects of DM on the heart and greatly reduce oxidative damage in myocardial cells. ${ }^{14,15}$ In light of the potential protective role of melatonin against myocardial damage, it is particularly noteworthy to explore whether melatonin interrupts the progression of DCM through modification of the mTOR signaling pathway and its downstream events. Therefore, this study was designed to discover the pathogenesis of the mTOR pathway in diabetes-induced cardiomyopathy and unveil the potential beneficial molecular basis of melatonin in DCM.

\section{Material and methods}

\section{Animal model design}

All the experimental procedures described in the study were performed in accordance with the Declaration of Helsinki and approved by Akdeniz University Institutional Animal Care and Use Committee Policies for Animal Use (Antalya, Turkey). As shown in Table 1, a total of 40 Wistar rats (8 weeks of age weighing 200-235 g) were randomly allocated into the following 4 groups; group 1 was the control group, group 2 was the vehicle group (50 cc/kg/day $10 \%$ ethanol injected group), group 3 was the DM group (iatrogenically DM-induced group), and group 4 was the melatonin + DM group (group given melatonin after iatrogenical DM induction) (Table 1).

\section{Induction of experimental diabetes mellitus type 1}

To induce experimental type I DM (pancreatic insulin release was blocked iatrogenically by affecting Langerhans islet cells), rats in group 3 and group 4 were injected intraperitoneally with a single dose of streptozotocin (STZ, $130 \mathrm{mg} / \mathrm{kg}$; Sigma-Aldrich, St. Louis, USA) in $0.1 \mathrm{~mol} / \mathrm{L}$ citrate buffer with a $\mathrm{pH}$ of 4.5. As described previously, successful induction of DM was defined as a constant blood glucose $>300 \mathrm{mg} / \mathrm{dL}$ in groups 3 and $4 . .^{16,17}$

\section{Transthoracic echocardiography and sample preparation}

Transthoracic echocardiography was performed in rats from all groups using the ultrasound system iE33 (Philips Medical Systems, Andover, USA) with a 1-3 MHz broadband linear array transducer at the induction of DM or citrate buffer injection and 8 weeks later. After the rats were anesthetized (with $50 \mathrm{mg} / \mathrm{kg}$ ketamine hydrochloride), the heart was imaged in the 2-dimensional parasternal short-axis view 3 times over at least 30 cardiac cycles to assess reproducibility. Mid-ventricle was recorded at the level of the papillary muscle using the M-mode echocardiography, and interventricular septal and posterior wall thickness (IVS and LVPW), as well as left ventricular end-diastolic and end-systolic internal diameters (LVEDD and LVESD) were determined. According to a well-established method,

Table 1. Definition of animal groups and applied drugs

\begin{tabular}{|l|c|c|c|}
\multicolumn{1}{|c|}{ Groups } & Injected drug & Dose & Period \\
\hline Group 1 (control) & none & none & none \\
\hline Group 2 (vehicle) & $10 \%$ ethanol & $50 \mathrm{cc} / \mathrm{kg} /$ day & 10 \\
\hline Group 3 (DM) & streptozotocin & $130 \mathrm{mg} / \mathrm{kg}$ & for 56 days \\
\hline Group 4 (melatonin + DM) & melatonin + streptozotocin & $50 \mathrm{mg} / \mathrm{kg} /$ day $+130 \mathrm{mg} / \mathrm{kg}$ & 10 \\
\hline
\end{tabular}

DM - diabetes mellitus. 
the left ventricular mass (LVM) was calculated with the $M$ mode (cubic) using the formula: LVM = 1.04[(IVS + LVID + LVPW $)^{3}-(\text { LVID })^{3}$ ], where 1.04 is the specific gravity of muscle. ${ }^{18}$ Left ventricle fractional shortening (FS) was used as an index of cardiac contractile function and was calculated from the inner diameters according to the formula: FS $(\%)=($ LVEDD - LVESD $) /$ LVEDD $\times 100$.

\section{Melatonin administration}

Melatonin (Sigma-Aldrich) was dissolved in absolute ethanol with a final concentration of $50 \mathrm{mg} / \mathrm{kg}$. It was daily injected intraperitoneally in the melatonin + DM group (group 4) for 56 days.

\section{Tissue preparation}

After the echocardiography animals were sacrificed. The hearts were carefully dissected and immersionfixed in a fixative containing $4 \%$ paraformaldehyde and $0.2 \%$ picric acid for $24 \mathrm{~h}$ at $4^{\circ} \mathrm{C}$. Then, the samples were washed in $0.1 \mathrm{M}$ phosphate-buffered saline (PBS), pH 7.4, for $24 \mathrm{~h}$ at $4^{\circ} \mathrm{C}$, cryoprotected with $30 \%$ saccharose and frozen-embedded in Tissue-Tek.

\section{Immunohistochemistry}

After incubating with rabbit antibodies against mTOR (1:500; Cell Signaling Tech., Beverly, USA) for $24 \mathrm{~h}$ at $4^{\circ} \mathrm{C}$, biotin-conjugated goat anti-rabbit IgG was added to the $10-\mu \mathrm{m}$ thick cryosections (1:300; Vector Lab., Peterborough, UK). Afterwards, the sections were exposed to avidin-biotin peroxidase complex (1:100; Elite ABC Kit, Vector Lab.) for $1 \mathrm{~h}$. The reaction occurred with $0.05 \%$ 3.3-diaminobenzidine tetra-hydrochloride (Sigma-Aldrich) in $0.05 \mathrm{M}$ Tris$\mathrm{HCl}$ buffer, $\mathrm{pH}$ 7.6, containing $0.01 \% \mathrm{H}_{2} \mathrm{O}_{2}$ and $0.01 \%$ nickel ammonium sulfate, as described previously. ${ }^{19}$ Incubations without the primary antisera served as controls.

\section{Western blot analysis}

Fifty micrograms of protein was loaded into each line on $10 \%$ SDS-PAGE (sodium-dodecyl-sulfate polycrylamide) gels. After electrophoresis, the proteins were electro-transferred to the polyvinylidene difluoride (PVDF) membrane (Bio-Rad Laboratories, Hercules, USA). The cells were electro-transferred to Immobilon-P transfer membrane (Merck Millipore, Billerica, USA) and Tris-buffered saline (TBS) that included 5\% non-fat milk for $1 \mathrm{~h}$ at room temperature. Then, the primary antibody was added and the samples were kept overnight at $4^{\circ} \mathrm{C}$, washed; then, a secondary antibody was used. The same procedure was repeated for beta-actin which was used as internal control.

\section{Measurements of superoxide dismutase, catalase and glutathione peroxidase activity, and malondialdehyde levels}

Heart samples were sonicated (Bandelin Sonopuls, HD 2070; Bandelin Electronic GmbH \& Co. KG, Berlin, Germany) in $500 \mu \mathrm{L}$ of ice-cold buffer $(50 \mathrm{mM}$ potassium phosphate, $\mathrm{pH}$ 7.0, $1 \mathrm{mM}$ ethylenediaminetetraacetic acid - EDTA), centrifuged (glutathione peroxidase (GPx) $-15,000 \mathrm{~g}$ for $10 \mathrm{~min}$ at $4^{\circ} \mathrm{C}$; catalase (CAT) $-10,000 \mathrm{~g}$ for $15 \mathrm{~min}$ at $4^{\circ} \mathrm{C}$; superoxide dismutase (SOD) - $1500 \mathrm{~g}$ for $5 \mathrm{~min}$ at $4^{\circ} \mathrm{C}$ ) and supernatants were collected and stored at $-80^{\circ} \mathrm{C}$ for later biochemical analysis according to established methods. ${ }^{20}$

Superoxide dismutase activity was measured using a SOD activity assay kit (Cayman Chemical, Ann Arbor, USA) in accordance with the methods described by Kaya et al. and Misra et al. ${ }^{20,21}$ An assay kit (Cayman-707002; Cayman Chemical) and spectrophotometric analysis were used to examine CAT enzymatic activity in the tissues in accordance with the methods described by Kaya et al. and Ziegler et al. ${ }^{20,22}$ Glutathione peroxidase activity was estimated indirectly using the coupled reaction with glutathione reductase assessed with GPx kit (SigmaAldrich Chemia, Steinheim, Germany), in accordance with the methods previously by Kaya et al. and Paglia et al. ${ }^{20,23}$ Malondialdehyde (MDA) levels were performed with a fluorometric method (as described in a previous paper) using 1.1.3.3-thtra-ethoxypropane as a standard. ${ }^{24}$

The protein concentrations were calculated spectrophotometrically (Shimadzu RF-5500; Shimadzu, Kyoto, Japan) using a protein assay reagent kit (Pierce, Rockford, USA), which is based on a modified Bradford method using bovine serine albumin as the standard.

\section{Quantification of staining intensities and statistical analysis}

For the densitometry analysis, a Zeiss Axioscop-2 Plus microscope at $\times 40$ magnification coupled with Image System Analysis, Axiovision v. 4.7 was used (Carl Zeiss, Jena, Germany). In a selected area, the average of the background color value was calculated. From the color measurement value of the heart muscle, this calculated average of the background gray value area was removed. Immunostaining intensity was presented as the mean of the measured heart muscle grey value minus the mean of the measured background grey value. Continuous variables were presented as mean + standard deviation (SD) for normal distributions. Normality of distribution was verified using the Shapiro-Wilk normality test. All continuous variables were distributing normally. All the data was presented as mean \pm SD. Paired samples t-test was performed to compare glucose levels. One-way analysis of variation (ANOVA) was performed after the assessment of distributions for more than 2 independent groups and 
a post hoc Dunnet test for unpaired data was applied to detect any differences between animal groups. SPSS software v. 21.0 for Windows (IBM Corp, Armonk, USA) was used for all statistical analyses. A p-value $<0.05$ was considered to indicate statistical significance.

\section{Results}

\section{Blood glucose levels}

The blood glucose levels are shown in Table 2. The blood glucose levels were found to be close to normal in all groups at the beginning of the study, but in animals that received STZ $130 \mathrm{mg} / \mathrm{kg}$, the plasma glucose level was significantly increased in both DM groups (group 3 and group 4$)$ at the end of the in vivo study $(\mathrm{p}<0.05)$ (Table 2$)$. Additionally, at the end of the study ( $56^{\text {th }}$ day), there was a statistically significant weight loss in group 3 compared to $1^{\text {st }}$ day (Table 3$)$.

\section{Effect of hyperglycemia on cardiac phenotype}

The hearts of animals in group 3 were hypertrophied and the ratio of the heart weight (W) and tibia length (L) as a marked of myocardial hypertrophy was significantly increased (W/L $[\mathrm{mg} / \mathrm{cm}]$ from $72 \pm 3$ to $90 \pm 8 ; \mathrm{p}<0.05)$. Echocardiography confirmed myocardial hypertrophy with protected systolic function in group 3, whereas echocardiographic data did not significantly change in group 4 (Table 3).

\section{The effect of melatonin treatment on expression of the mammalian target of rapamycin in heart muscle fibers shown with immunochemistry}

The staining intensities of the expression of the mTOR levels in the diabetic group (group 3) were higher than in the control group (914.9 vs 510.7, $\mathrm{p}<0.05$ ). There was a difference of $79.2 \%$ between the control group (group 1) and the DM group (group 3). In the melatonin + DM group (group 4), the staining intensities of the expression of mTOR levels were significantly lower compared to the DM group (group 3), and this differences was $32.2 \%$ (620.3 vs 914.9, $\mathrm{p}<0.05)$. No significant difference in the staining intensities of the expression of mTOR levels was found between the rats in groups 1 and 2 (510.7 vs 520.4, $\mathrm{p}>0.05$ ) (Fig. 1).

\section{The mammalian target of rapamycin in heart muscle fibers shown with western blot}

The mTOR expression levels were measured in the rat heart muscle fibers in all groups and all data is shown in Fig. 2. The mTOR expression in rats treated with

Table 2. Blood glucose levels of group 3 (DM) and group 4 (melatonin + DM) post streptozotocin injection

\begin{tabular}{|l|c|c|c|c|} 
& $\begin{array}{c}3^{\text {rd }} \text { day } \\
\text { post injection } \\
(\mathrm{mg} / \mathrm{dL})\end{array}$ & $\begin{array}{c}14^{\text {th }} \text { day } \\
\text { post injection } \\
(\mathrm{mg} / \mathrm{dL})\end{array}$ & $\begin{array}{c}2^{\text {th }} \text { day } \\
\text { post injection } \\
(\mathrm{mg} / \mathrm{dL})\end{array}$ & $\begin{array}{c}42^{\text {th }} \text { day } \\
\text { post injection } \\
(\mathrm{mg} / \mathrm{dL})\end{array}$ \\
\hline Group 3 & $354 \pm 18$ & $367 \pm 14$ & $413 \pm 14$ & $417 \pm 17$ \\
\hline Group 4 & $347 \pm 12$ & $363 \pm 21$ & $402 \pm 17$ & $417 \pm 12^{*}$ \\
\hline
\end{tabular}

DM - diabetes mellitus. Data is presented as mean \pm standard deviation (SD). ${ }^{*}$ indicates a p-value $<0.05$ compared with the $3^{\text {rd }}$ day of injection.

Table 3. Systolic and diastolic echocardiographic measurements of the groups

\begin{tabular}{|c|c|c|c|c|c|}
\hline \multicolumn{2}{|c|}{ Variables } & $\begin{array}{c}\text { Group } 1 \\
n=10\end{array}$ & $\begin{array}{c}\text { Group } 2 \\
n=10\end{array}$ & $\begin{array}{c}\text { Group } 3 \\
n=10\end{array}$ & $\begin{array}{c}\text { Group } 4 \\
n=10\end{array}$ \\
\hline \multirow{3}{*}{ Systolic } & septum [mm] & $1.43 \pm 0.03$ & $1.45 \pm 0.06$ & $1.97 \pm 0.03^{*}$ & $1.39 \pm 0.13$ \\
\hline & LVESD [mm] & $1.50 \pm 0.06$ & $1.63 \pm 0.30$ & $1.43 \pm 0.15^{*}$ & $1.57 \pm 0.10$ \\
\hline & LVPWD [mm] & $1.21 \pm 0.41$ & $1.24 \pm 0.08$ & $1.79 \pm 0.02^{*}$ & $1.33 \pm 0.04$ \\
\hline \multirow{6}{*}{ Diastolic } & septum [mm] & $0.68 \pm 0.40$ & $0.71 \pm 0.01$ & $1.05 \pm 0.05^{*}$ & $0.79 \pm 0.05$ \\
\hline & LVEDD [mm] & $2.97 \pm 0.30$ & $3.01 \pm 0.24$ & $3.96 \pm 0.12^{*}$ & $3.02 \pm 0.01$ \\
\hline & LVPWD [mm] & $0.82 \pm 0.03$ & $0.80 \pm 0.04$ & $1.17 \pm 0.06^{*}$ & $0.79 \pm 0.02$ \\
\hline & LVM [mg] & $98 \pm 10$ & $106 \pm 7$ & $137 \pm 18^{*}$ & $104 \pm 11$ \\
\hline & FS [\%] & $54 \pm 1$ & $44 \pm 3$ & $49 \pm 3$ & $51 \pm 7$ \\
\hline & heart rate $[\mathrm{bpm} / \mathrm{min}]$ & $479 \pm 12$ & $475 \pm 12$ & $476 \pm 19$ & $474 \pm 19$ \\
\hline \multirow{2}{*}{ Body weight } & $1^{\text {st }}$ day $[g]$ & $213.70 \pm 8.32$ & $219.50 \pm 6.36$ & $223.80 \pm 8.39$ & $217.60 \pm 7.87$ \\
\hline & $56^{\text {th }}$ day $[g]$ & $215.60 \pm 7.52$ & $218.60 \pm 9.42$ & $191.30 \pm 10.80^{*}$ & $210.60 \pm 9.65$ \\
\hline
\end{tabular}

LVESD - left ventricular end systolic diameter; LVPWD - left ventricular posterior wall diameter; LVEDD - left ventricular end diastolic diameter; LVM - left ventricular mass; FS - fractional shortening. Data is presented as mean \pm standard deviation (SD). * indicates a p-value $<0.05$ compared with control group. 

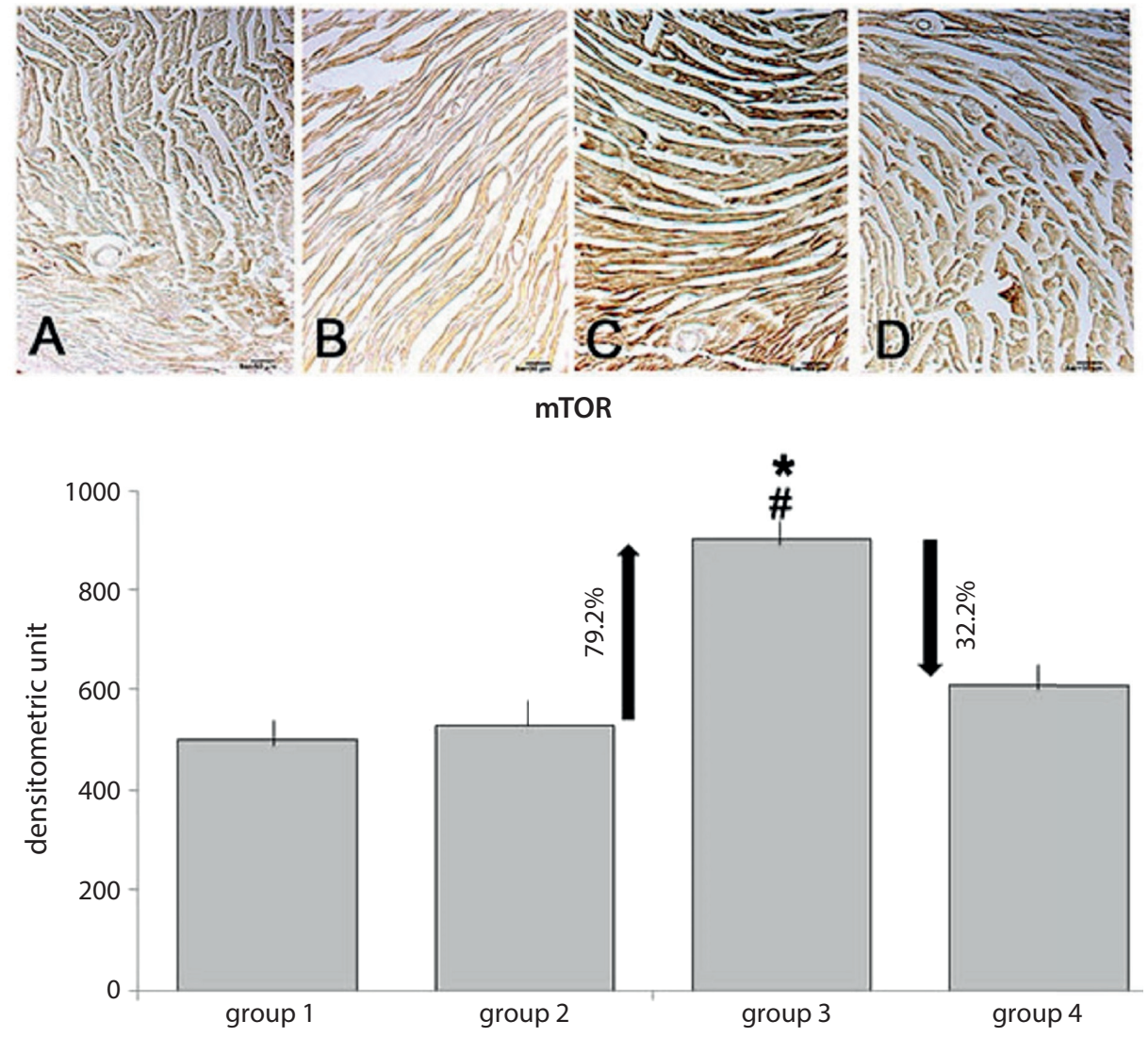

Fig. 1. Expression and densitometry analysis of the mammalian target of rapamycin (mTOR) in heart muscle fibers shown using immunohistochemistry. The staining intensities of the expression of the MTOR levels of the control group (group 1) (A), vehicle group (group 2) (B), diabetes mellitus (DM) group (group 3) (C), and melatonin + DM group (group 4) (D). Data was presented as mean \pm standard deviation (SD); $n=10$ for each group

* indicates statistically significant difference between DM group (group 3) and control group (group 1); \# indicates statistically significant difference between the DM group (group 3) and melatonin + DM group (group 4); $p<0.05$; bar: $50 \mu \mathrm{m}$.
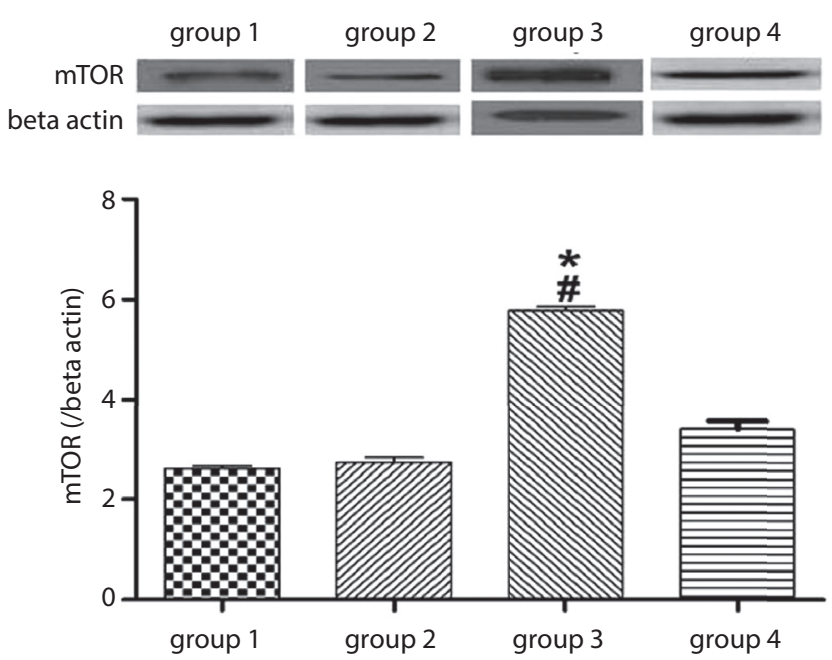

Fig. 2. The mammalian target of rapamycin (mTOR) levels in heart muscle fibers shown using western blot. The mTOR levels of the control (group 1), vehicle (group 2), diabetes mellitus (group 3), and melatonin + DM (group 4). Beta-actin was used for internal control. Data was presented as mean \pm standard deviation (SD); $n=10$ for each group

* indicates statistically significant difference between DM group (group 3) and control (group 1); \# indicates statistically significant difference between DM group (group 3) and melatonin + DM group (group 4); $\mathrm{p}<0.05$; bar: $50 \mu \mathrm{m}$.

melatonin (group 4) was lower than in DM group (group 3) $(\mathrm{p}<0.05)$. No difference was observed between the control group (group 1) and the vehicle group (group 2) (Fig. 2).

\section{Biochemical analysis}

All 3 antioxidant enzymes (CAT, SOD and GPx) activities in heart fibers under diabetic conditions under melatonin treatment were measured. The serum antioxidant enzymes activities (SOD, CAT and GPx) were significantly lower in the DM group (group 3) than in the control group (group 1) (for all p < 0.05), whereas compared to the control, $50 \mathrm{mg} / \mathrm{kg}$ melatonin treatment resulted in a significant increase in the activities of antioxidant enzymes under hyperglycemic conditions. Additionally, it was shown that melatonin treatment protected the antioxidant activity in heart fibers under hyperglycemic conditions against the hypertrophy (Fig. 3).

\section{Discussion}

In this study, 4 important results have been elicited. Firstly, the diabetic conditions triggered the development of cardiac hypertrophy. Secondly, mTOR expression increased in the diabetic setting is activated by high glucose and contributes to the development of DCM. Thirdly, melatonin treatment prevented the development of cardiac hypertrophy in hyperglycemia. Finally, melatonin treatment increased the activity of the antioxidant enzyme, which was reduced by the effect of DM. 

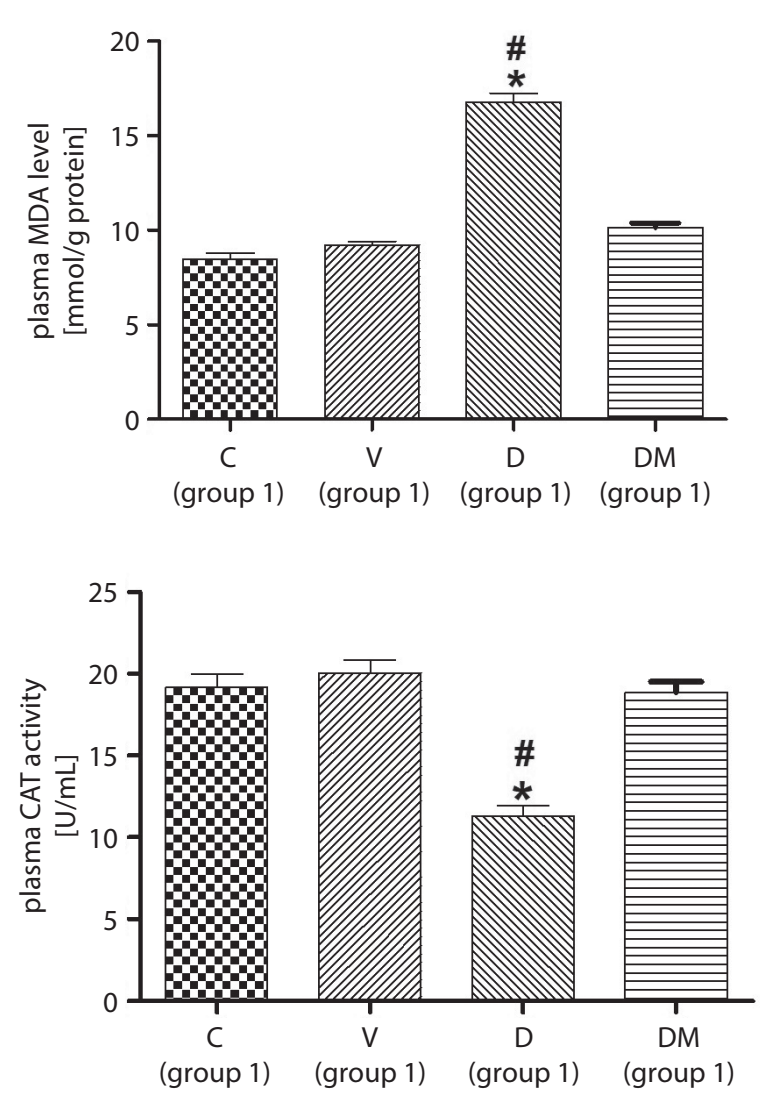
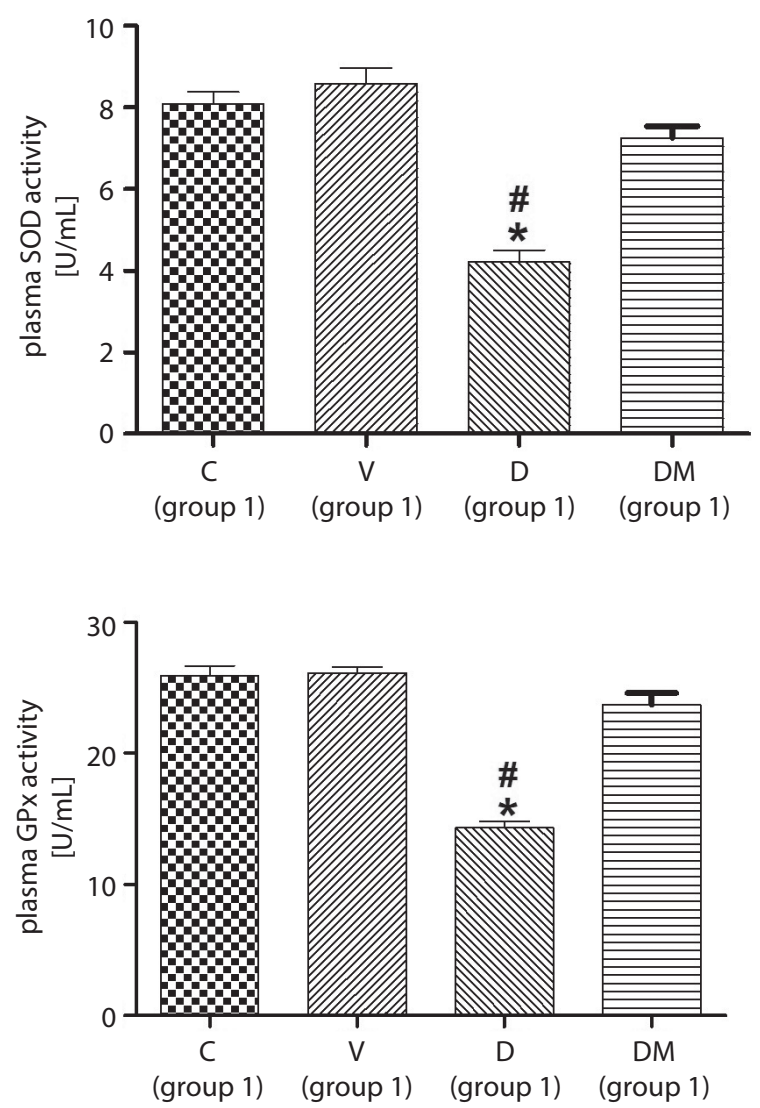

Fig. 3. Malondialdehyde (MDA) levels and antioxidant enzymes (glutathione peroxidase (GPX), catalase (CAT) and superoxide dismutase (SOD)) activities between groups. Diagram shows GPx, CAT and SOD enzymes activities and MDA levels in plasma samples of animal groups. Note that GPx, CAT and SOD activities were significantly lower in the DM group (group 3) than in the control group (group 1), and MDA levels were significantly higher in the DM group (group 3)

* indicates statistically significant difference between DM group (group 3) and control group (group 1); \# indicates statistically significant difference between DM group (group 3) and melatonin + DM group (group 4); p < 0.05; bar: $50 \mu \mathrm{m}$.

Diabetic cardiomyopathy is a clinical condition diagnosed when ventricular dysfunction occurs in the absence of coronary atherosclerosis and hypertension according to the cardiology guidelines. ${ }^{25}$ Diabetic cardiomyopathy observed in insulin-resistant or hyperinsulinemic states is characterized by impaired myocardial insulin signaling, mitochondrial dysfunction, endoplasmic reticulum stress, impaired calcium homeostasis, abnormal coronary microcirculation, activation of renin-angiotensin-aldosterone system, sympathetic nervous system, and maladaptive immune responses. ${ }^{26}$ These pathophysiological changes lead to multiple toxic effects on cardiomyocytes. The mTOR is an important protein modulator of the insulin-signaling pathway. It was previously demonstrated that in DM, chronically activated mTOR induces multiple pathological events, including a negative feedback loop that suppresses insulin receptor substrate. The mTOR signaling pathway is correlated to the occurrence of both cardiomyopathy and cardiac hypertrophy. At the same time, researchers have shown that mTOR plays an important role in the cardiac hypertrophy that occurs in STZ-induced experimental DM. Moreover, short-term treatment with rapamycin, a mTOR inhibitor, was a promising strategy for cardiac diseases such as acute myocardial infarctions and cardiac hypertrophy in DM. .,8,27-29 $^{\text {. }}$

In addition, it has been emphasized that melatonin treatment is the mediator of mTOR activation. For example, in one study, it was found that melatonin treatment reduces mTOR activation, which reduces cell viability and proliferation. ${ }^{30}$ Our data supports the functional significance of mTOR in diabetic cardiac hypertrophy. In our study, we also provided an effective method of suppressing mTOR with melatonin treatment. This study is the first to demonstrate the molecule pathway of the effect of melatonin on diabetic cardiac hypertrophy by decreasing mTOR. In parallel with the literature, we propose a new pathway which higher glucose level stimulus induces diabetic cardiomyopathy through the activation of mTOR, and this pathway could be eradicated by melatonin treatment.

Anti-oxidative enzymes (SOD, CAT and GPx), which metabolize radicals or reactive oxygen species (ROS) into non-radical products, play a crucial role in antioxidant defense. ${ }^{31}$ In different studies, it has been established that melatonin treatment increases the activity of antioxidant enzymes, such as SOD, CAT and GPx in different tissues in which oxidative stress was induced by various agents. ${ }^{32,33}$ 
It was proposed by several studies that high levels of oxidative stress are related to apoptosis of myocardial cells and the pathological process that causes cardiac hypertrophy. ${ }^{34}$ It was shown in the current study that MDA levels were increased with DM, and this situation is indicating that high stress from ROS is involved in the pathological development of diabetic cardiomyopathy. Additionally, the melatonin + DM group (group 4) had remarkably increased antioxidant enzymes activities and significantly decreased MDA levels, which is a marker of oxidative stress compared to the DM group (group 3).

In conclusion, our data shows that the cardioprotective effect of melatonin caused by inhibiting mTOR expression may prevent cardiac hypertrophy. Demonstrating the underlying mechanism behind mTOR-mediated pathophysiological features in the heart will be important in improving therapies of DCM concerning melatonin.

\section{ORCID iDs}

Unal Güntekin (1) https://orcid.org/0000-0002-1344-6250

Veysel Tosun (10 https://orcid.org/0000-0001-7629-2108

Yasemin Behram Kandemir (10) https://orcid.org/0000-0002-6490-9155

\section{References}

1. Levy D, Garrison RJ, Savage DD, Kannel WB, Castelli WP. Prognostic implications of echocardiographically determined left ventricular mass in the Framingham Heart Study. N Engl J Med. 1990;322(22):1561-1566.

2. Marwick TH, Ritchie R, Shaw JE, Kaye D. Implications of underlying mechanisms for the recognition and management of diabetic cardiomyopathy. J Am Coll Cardiol. 2018;71(3):339-351.

3. Tsushima $\mathrm{K}$, Bugger $\mathrm{H}$, Wende AR, et al. Mitochondrial reactive oxygen species in lipotoxic hearts induces post-translational modifications of AKAP121, DRP1 and OPA1 that promote mitochondrial fission. Circ Res. 2018;122(1):58-73.

4. Liu W, Ruiz-Velasco A, Wang S, et al. Metabolic stress-induced cardiomyopathy is caused by mitochondrial dysfunction due to attenuated Erk5 signaling. Nat Commun. 2017;8(1):494.

5. Zhang Y, Sun X, Icli B, Feinberg MW. Emerging roles for microRNAs in diabetic microvascular disease: Novel targets for therapy. Endocr Rev. 2017;38(2):145-168.

6. Shimobayashi M, Hall MN. Making new contacts: the mTOR network in metabolism and signaling crosstalk. Nat Rev Mol Cell Biol. 2014; 15(3):155.

7. Shende P, Plaisance I, Morandi C, et al. Cardiac raptor ablation impairs adaptive hypertrophy, alters metabolic gene expression, and causes heart failure in mice. Circulation. 2011;123(10):1073-1082.

8. Aoyagi T, Kusakari Y, Xiao CY, et al. Cardiac mTOR protects the heart against ischemia-reperfusion injury. Am J Physiol Heart Circ Physiol. 2012;303(1):H75-H85.

9. Huang Z, Zhuang X, Xie C, et al. Exogenous hydrogen sulfide attenuates high glucose-induced cardiotoxicity by inhibiting NLRP3 inflammasome activation by suppressing TLR4/NF-KB pathway in $\mathrm{H} 9 \mathrm{c} 2$ cells. Cell Physiol Biochem. 2016;40(6):1578-1590.

10. Patel V, Upaganlawar A, Zalawadia R, Balaraman R. Cardioprotective effect of melatonin against isoproterenol induced myocardial infarction in rats: A biochemical, electrocardiographic and histoarchitectural evaluation. Eur J Pharmacol. 2010;644(1-3):160-168.

11. Anderson EJ, Kypson AP, Rodriguez E, Anderson CA, Lehr EJ, Neufer PD. Substrate-specific derangements in mitochondrial metabolism and redox balance in the atrium of the type 2 diabetic human heart. J Am Coll Cardiol. 2009;54(20):1891-1898.

12. Braunwald E. Biomarkers in heart failure. N Engl J Med. 2008;358(20): 2148-2159.

13. Zhao F, Liu ZQ, Wu D. Antioxidative effect of melatonin on DNA and erythrocytes against free-radical-induced oxidation. Chem Phys Lipids. 2008;151(2):77-84.
14. Lin XJ, Mei GP, Liu J, et al. Therapeutic effects of melatonin on heatstroke-induced multiple organ dysfunction syndrome in rats. J Pineal Res. 2011;50(4):436-444.

15. Liu LF, Qin Q, Qian ZH, et al. Protective effects of melatonin on ischemia-reperfusion induced myocardial damage and hemodynamic recovery in rats. Eur Rev Med Pharmacol Sci. 2014;18(23):3681-3686.

16. Hoyer DP, Korkmaz Y, Grönke S, et al. Differential expression of protein kinase $C$ isoforms in coronary arteries of diabetic mice lacking the G-protein Ga11. Cardiovasc Diabetol. 2010;9:93.

17. Reuter H, Seuthe K, Korkmaz Y, et al. The G protein Ga11 is essential for hypertrophic signaling in diabetic myocardium. Int J Cardiol. 2013;167(4):1476-1485

18. Devereux RB, Reichek N. Echocardiographic determination of left ventricular mass in man. Anatomic validation of the method. Circulation. 1977;55(4):613-618.

19. Korkmaz $\mathrm{Y}$, Lang $\mathrm{H}$, Beikler T, et al. Irreversible inflammation is associated with decreased levels of the a1-, $\beta 1-$, and a2-subunits of sGC in human odontoblasts. J Dent Res. 2011;90(4):517-522.

20. Kaya Y, Sarıkcıoğlu L, Aslan M, et al. Comparison of the beneficial effect of melatonin on recovery after cut and crush sciatic nerve injury: a combined study using functional, electrophysiological, biochemical, and electron microscopic analyses. Childs Nerv Syst. 2013;29(3):389-401.

21. Misra HP, Fridovich I. The role of superoxide anion in the autoxidation of epinephrine and a simple assay for superoxide dismutase. J Biol Chem. 1972;247(10):3170-3175.

22. Ziegler DM, Kehrer JP. Oxygen radicals and drugs: In vitro measurements. Methods Enzymol. 1990;186:621-626.

23. Paglia DE, Valentine WN. Studies on the quantitative and qualitative characterization of erythrocyte glutathione peroxidase. J Lab Clin Med. 1967;70(1):158-169.

24. Wasowicz W, Neve J, Peretz A. Optimized steps in fluorometric determination of thiobarbituric acid-reactive substances in serum: Importance of extraction $\mathrm{pH}$ and influence of sample preservation and storage. Clin Chem. 1993;39(12):2522-2526.

25. Rydén L, Grant PJ, Anker SD, et al; Authors/Task Force Members. ESC Guidelines on diabetes, pre-diabetes, and cardiovascular diseases developed in collaboration with the EASD: The Task Force on diabetes, pre-diabetes, and cardiovascular diseases of the European Society of Cardiology (ESC) and developed in collaboration with the European Association for the Study of Diabetes (EASD). Eur Heart J. 2013;34(39):3035-3087.

26. Jia G, DeMarco VG, Sowers JR. Insulin resistance and hyperinsulinemia in diabetic cardiomyopathy. Nat Rev Endocrinol. 2016;12(3):144-153.

27. Pei Z, Deng Q, Babcock SA, He EY, Ren J, Zhang Y. Inhibition of advanced glycation endproduct (AGE) rescues against streptozotocin-induced diabetic cardiomyopathy: Role of autophagy and ER stress. Toxicol Lett. 2018;284:10-20.

28. Suhara T, Baba Y, Shimada BK, Higa JK, Matsui T. The mTOR signaling pathway in myocardial dysfunction in type 2 diabetes mellitus. Curr Diab Rep. 2017;17(6):38.

29. Zhang $P, X u X, H u X$, Van Deel ED, Zhu G, Chen Y. Inducible nitric oxide synthase deficiency protects the heart from systolic overloadinduced ventricular hypertrophy and congestive heart failure. Circ Res. 2007;100(7):1089-1098.

30. Shen YQ, Guerra-Librero A, Fernandez-Gil BI, et al. Combination of melatonin and rapamycin for head and neck cancer therapy: Suppression of AKT/mTOR pathway activation, and activation of mitophagy and apoptosis via mitochondrial function regulation. J Pineal Res. 2018;63(3). https://doi.org/10.1111/jpi.12461

31. Baydas G, Canatan H, Turkoglu A. Comparative analysis of the protective effects of melatonin and vitamin E on streptozocin-induced diabetes mellitus. J Pineal Res. 2002;32(4):225-230.

32. Ohta Y, Kongo-Nishimura M, Matsura T, Yamada K, Kitagawa A, Kishikawa T. Melatonin prevents disruption of hepatic reactive oxygen species metabolism in rats treated with carbon tetrachloride. JPineal Res. 2004;36(1):10-17.

33. Sailaja Devi MM, Suresh Y, Das UN. Preservation of the antioxidant status in chemically induced diabetes mellitus by melatonin. J Pineal Res. 2000;29(2):108-115.

34. Song E, Jahng JW, Chong LP, et al. Lipocalin-2 induces NLRP3 inflammasome activation via HMGB1 induced TLR4 signaling in heart tissue of mice under pressure overload challenge. Am JTransI Res. 2017;9(6):2723-2735. 